\title{
CRISPRing or RISKing? Dangers Arising from Gene Editing with CRISPR-Cas9
}

\author{
Antonis Giakountis ${ }^{1,2 *}$ \\ ${ }^{1}$ Department of Biochemistry and Biotechnology, University of Thessaly, Greece \\ 2Department of Molecular Biology and Genetics, Biomedical Sciences Research Center \\ "Alexander Fleming”, Greece
}

Editorial

Volume 2 Issue 2

Received Date: July 04, 2018

Published Date: July 10, 2018

*Corresponding author: Antonis Giakountis, Department of Biochemistry and Biotechnology, University of Thessaly, 41500 Larisa, Greece, Tel: +30 2410-565206; Email: giakountis@fleming.gr

\section{Editorial}

Since its first experimental demonstration in 2007 [1] CRISPR-Cas9 (short for Clustered Regularly Interspaced Short Palindromic Repeats - CRISPR-Associated protein 9) brought a revolution in biology, especially in the fields of gene editing [2] and precision medicine [3]. CRISPR offers several advantages, namely accuracy [4], flexibility [5], versatility [6] and cost reduction [7] that collectively designate it as the most popular approach for gene editing [8], supplanting in parallel other technologies such as TALENs [9] and ZFNs [10].

Similar to TALEN/ZNFs, CRISPR-Cas9 relies on the precise introduction of DSBs (Double Strand Breaks) in the target genome [11], facilitated by the interaction of complementary small RNAs, known as sgRNAs (Single Guide RNAs) with the Cas9 effector protein [12]. The sgRNA/Cas9 ribonucleoprotein complex interacts with genomic DNA via sgRNA/genomic DNA R-loop formation $[13,14]$, guiding wild type Cas9 to introduce DSBs via its nuclease activity in a precise manner [15]. Host genome DNA repair mechanisms, such as Non-Homologous-End Joining (NHEJ) and/or Homology-Directed Repair (HDR) recognise and repair DBS formation, resulting either in the introduction of mutating Insertions/Deletions (InDels) in the case of NHEJ [16], or precision gene editing and Single Nucleotide Polymorphism (SNP) repair in the case of HDR [17].

Targeted mutations can restrict or completely abolish cas9 nuclease activity, converting it into a nickase (nCas9, $[18,19]$ ) or a catalytically inactive protein (dCas9, [20]) that retains its DNA-interacting properties. These engineered flavours of Cas9 contribute to the reduction of its greater side-effect, generation of off-target effects [21], while expanding the repertoire of CRISPR applications from a mere gene editing tool to a technology that allows in vivo biotagging [22] and/or transcriptional transactivation/repression through direct endogenous promoter interactions [23].

Applications of CRISPR-Cas9 greatly expanded our knowledge in various organisms such as bacteria [24], yeast [25], worms [26], insects [27], plants [28] and animals [29-32], fuelling an active debate as to whether this technology is safe enough for human gene editing clinical trials [33]. The benefits are obvious: precision medicines, treatment of hereditary disease, stalling/curing of cancer progression [34], are just a few of the exciting opportunities arising from the technology [35].

However two recent papers published in Nature Medicine highlight the important biases and dangers that accompany CRISPR-Cas9 mediated editing in human cells. The first paper refers to a CRISPR-Cas9 dropout screen in retinal pigment epithelium cells (RPE1) [36]. Such screens rely on a lentiviral pool of sgRNAs, designed to target hundreds to thousands of genes, for transducing Cas9expressing cells. The successfully transduced cells are left to propagate for a limited period before being subjected to Next Generation Sequencing (NGS)-based screening. The concept is obvious: sgRNAs targeting genes that are pivotal for the survival of cells under the screening conditions will be eliminated from the pool resulting into 


\section{Medicinal \& Analytical Chemistry International Journal}

a detectable distortion in the composition of sgRNA expressing cells in the NGS results, leading towards the identification of the corresponding target genes [37].

The authors report that Cas9-induced DSS activate p53, a well known tumor-suppressor that acts as a master regulator of DNA damage response [38], leading to cell cycle arrest and introduction of a growth disadvantage that eventually shifts the balance from HDR (precision editing) to NHEJ (imperfect repair/introduction of InDels) [36]. The growth defect was chemically dampened with the use of nutlin-3a, an inhibitor of p53 [36]. However general inhibition of p53 via nutlin-3a leaves the recipient cell vulnerable to major genetic abrogations, such as chromosome rearrangement/loss, accumulation of mutations and malignant transformation, renting this approach unsuited for safe therapeutic use of CRISPRCas9 mediated gene editing.

In a parallel study, scientists from Novartis conducted genetics screens in human pluripotent stem cells (hPSCs). Despite of their great therapeutic potential, screens in hPSCs have been impeded on the generally low efficiency of these cells to genetic engineering [39]. CRISPR-based editing offers great precision and together with the pluripotency of hPSCs provides an attractive therapeutic tool. Strikingly, the increased mutagenic efficiency of CRISPR in the hPSCs in the case of the Novartis study was accompanied with severe toxicity leading to a dramatic decrease in the percentage of surviving cells [39]. This toxicity was tightly linked to the generation of DSBs that were induced by targeting sgRNAs, persisted even under short induction of Cas9 expression and was not observed in hPSCs transduced with scrambled sgRNAs [39]. Additional experiments narrowed down the cause of this DSB-induced toxicity again to a P53-depended action [39].

These two studies collectively highlight the risk of deploying CRISPR-Cas9 as a therapeutic gene editing tool, nailing down the cause of the observed toxicity into the role of the p53 tumor-suppressor gene. CRISPR-Cas9 holds a great therapeutic potential, calling for a need to develop novel chemical and/or genetic approaches so as to eliminate the observed p53 complications. Our designing efforts should not focus on the general inhibition of p53 since this would increase the tumorigenicity of the engineered cell. Instead we should develop compounds that transiently block p53 function and/or block Cas 9 mediated genotoxic stress ensuring both efficient gene editing and subsequent p53 antitumorigenic protection for the engineered human stem cell.

\section{References}

1. Barrangou R, Fremaux C, Deveau H, Richards M, Boyaval P, et al. (2007) CRISPR provides acquired resistance against viruses in prokaryotes. Science 315(5819): 1709-1712.

2. Sander JD, Joung JK (2014) CRISPR-Cas systems for editing, regulating and targeting genomes. Nat Biotechnol 32(4): 347-355.

3. Precision ST (2017) Medicine, CRISPR, and Genome Engineering. Advances in Experimental Medicine and Biology, TS: Springer International Publishing, pp: 1178.

4. Chen JS, Dagdas YS, Kleinstiver BP, Welch MM, Sousa AA, et al. (2017) Enhanced proofreading governs CRISPR-Cas9 targeting accuracy. Nature 550(7676): 407-410.

5. Zhou Y, Zhu S, Cai C, Yuan P, Li C, et al. (2014) Highthroughput screening of a CRISPR/Cas9 library for functional genomics in human cells. Nature 509(7501): 487-491.

6. Wojtal D, Kamaladewi DU, Malam Z, Abdullah S, Wong TW, et al. (2016) Spell Checking Nature: Versatility of CRISPR/Cas9 for Developing Treatments for Inherited Disorders. Am J Hum Genet 98(1): 90-101.

7. Liu ET, Bolcun-Filas E, Grass DS, Lutz C, Murray S, et al. (2017) Of mice and CRISPR: The post-CRISPR future of the mouse as a model system for the human condition. EMBO Rep 18(2): 187-193.

8. Pennisi E (2013) The CRISPR craze. Science 341(6148): 833-836.

9. Joung JK, Sander JD (2013) TALENs: a widely applicable technology for targeted genome editing. Nat Rev Mol Cell Biol 14(1): 49-55.

10. Carroll D (2011) Genome engineering with zincfinger nucleases. Genetics 188(4): 773-782.

11. Ran FA, Hsu PD, Wright J, Agarwala V, Scott DA, et al. (2013) Genome engineering using the CRISPR-Cas9 system. Nat Protoc 8(11): 2281-2308.

12. Nishimasu H, Ran FA, Hsu PD, Konermann S, Shehata SI, et al. (2014) Crystal structure of Cas9 in complex with guide RNA and target DNA. Cell 156(5): 935-949. 


\section{Medicinal \& Analytical Chemistry International Journal}

13. Lim Y, Bak YS, Sung K, Jeong E, Lee SH, et al. (2016) Structural roles of guide RNAs in the nuclease activity of Cas9 endonuclease. Nat Commun 7: 13350.

14. Rutkauskas M, Sinkunas T, Songailiene I, Tikhomirova MS, Siksnys V, et al. (2015) Directional R-Loop Formation by the CRISPR-Cas Surveillance Complex Cascade Provides Efficient Off-Target Site Rejection. Cell Rep 2211-1247(15): 00135-00137.

15. Salsman J, Dellaire G (2017) Precision genome editing in the CRISPR era. Biochem Cell Biol 95(2): 187-201.

16. Su T, Liu F, Gu P, Jin H, Chang Y, et al. (2016) A CRISPR-Cas9 Assisted Non-Homologous End-Joining Strategy for One-step Engineering of Bacterial Genome. Sci Rep 6: 37895.

17. Kan Y, Ruis B, Takasugi T, Hendrickson EA (2017) Mechanisms of precise genome editing using oligonucleotide donors. Genome Res 27(7): 10991111.

18. Ran FA, Hsu PD, Lin CY, Gootenberg JS, Konermann S, et al. (2013) Double nicking by RNA-guided CRISPR Cas9 for enhanced genome editing specificity. Cell 154(6): 1380-1389.

19. Chiang TW, Ie Sege C, Larrieu D, Demir M, Jackson SP (2016) CRISPR-Cas9(D10A) nickase-based genotypic and phenotypic screening to enhance genome editing. Sci Rep 6: 24356.

20. Qi LS, Larson MH, Gilbert LA, Doudna JA, Weissman JS, et al. (2013) Repurposing CRISPR as an RNAguided platform for sequence-specific control of gene expression. Cell 152(5): 1173-1183.

21. Hsu PD, Scott DA, Weinstein JA, Ran FA, Konermann S, et al. (2013) DNA targeting specificity of RNA-guided Cas9 nucleases. Nat Biotechnol 31(9): 827-832.

22. Ye H, Rong Z, Lin Y (2017) Live cell imaging of genomic loci using dCas9-SunTag system and a bright fluorescent protein. Protein Cell 8(11): 853-855.

23. Perez-Pinera P, Kocak DD, Vockley CM, Adler AF, Kabadi AM, et al. (2013) RNA-guided gene activation by CRISPR-Cas9-based transcription factors. Nat Methods 10(10): 973-976.

24. Cho S, Shin J, Cho BK (2018) Applications of CRISPR/Cas System to Bacterial Metabolic Engineering. Int J Mol Sci 19(4).
25. Giersch RM, Finnigan GC (2017) Yeast Still a Beast: Diverse Applications of CRISPR/Cas Editing Technology in S. cerevisiae. Yale J Biol Med 90(4): 643-651.

26. Xu S (2015) The application of CRISPR-Cas9 genome editing in Caenorhabditis elegans. J Genet Genomics 42(8): 413-421.

27. Taning CNT, Van Eyande B, Yu N, Ma S, Smagghe G (2017) CRISPR/Cas9 in insects: Applications, best practices and biosafety concerns. J Insect Physiol 98: 245-257.

28. Liu X, Wu S, Xu J, Sui C, Wei J (2017) Application of CRISPR/Cas9 in plant biology. Acta Pharm Sin B 7(3): 292-302.

29. Maeder ML, Linder SJ, Cascio VM, Fu Y, Ho QH, et al. (2013) CRISPR RNA-guided activation of endogenous human genes. Nat Methods 10(10): 977-979.

30. Shalem O, Sanjana NE, Hartenian E, Shi X, Scott DA, et al. (2014) Genome-scale CRISPR-Cas9 knockout screening in human cells. Science 343(6166): 84-87.

31. Wang H, Yang H, Shivalila CS, Dawlaty MM, Cheng AW, et al. (2013) One-step generation of mice carrying mutations in multiple genes by CRISPR/Cas-mediated genome engineering. Cell 153(4): 910-118.

32. Yoshimi K, Kaneko T, Voigt B, Mashimo T (2014) Allele-specific genome editing and correction of disease-associated phenotypes in rats using the CRISPR-Cas platform. Nat Commun 5: 4240.

33. Eid A, Mahfouz MM (2016) Genome editing: the road of CRISPR/Cas9 from bench to clinic. Exp Mol Med 48(10): e265.

34. Zhan T, Rindtorff N, Betge J, Ebert MP, Boutros M (2018) CRISPR/Cas9 for cancer research and therapy. Semin Cancer Biol 1044-1579(17): 30274-30272.

35. Savic N, Schwank G (2016) Advances in therapeutic CRISPR/Cas9 genome editing. Transl Res 168: 15-21.

36. Haapaniemi E, Botla S, Persson J, Schmierer B, Taipale J (2018) CRISPR-Cas9 genome editing induces a p53mediated DNA damage response. Nat Med

37. Schmierer B, Botla SK, Zhang J, Turunen M, Kivioja T, et al. (2017) CRISPR/Cas9 screening using unique molecular identifiers. Mol Syst Biol 13(10): 945. 


\section{Medicinal \& Analytical Chemistry International Journal}

38. Joerger AC, Fersht AR (2016) The p53 Pathway: Origins, Inactivation in Cancer, and Emerging Therapeutic Approaches. Annu Rev Biochem 85: 375404.
39. Ihry RJ, Worringer KA, Salick MR, Frias M, Ho D, et al. (2018) p53 inhibits CRISPR-Cas9 engineering in human pluripotent stem cells. Nat Med.

\section{(c) (i)}

